ESTE

ESTE

Facultad de CC. EE. Ekonomia eta Enpresa y Empresariales

Zientzien Fakultatea

\title{
EL MÉTODO DELPHI
}

\section{Eneko Astigarraga}

Universidad de Deusto

Facultad de CC.EE. y Empresariales. ESTE

Mundaiz, 50

Apartado 1.359

E-20.080 Donostia - San Sebastian 
ESTE

\section{EL MÉTODO DELPHI}

El método Delphi1, cuyo nombre se inspira en el antiguo oráculo de Delphos, parece que fue ideado originalmente a comienzos de los años 50 en el seno del Centro de Investigación estadounidense RAND Corporation por Olaf Helmer y Theodore J. Gordon, como un instrumento para realizar predicciones sobre un caso de catástrofe nuclear. Desde entonces, ha sido utilizado frecuentemente como sistema para obtener información sobre el futuro.

Linston y Turoff 2 definen la técnica Delphi como un método de estructuración de un proceso de comunicación grupal que es efectivo a la hora de permitir a un grupo de individuos, como un todo, tratar un problema complejo.

Una Delphi consiste en la selección de un grupo de expertos a los que se les pregunta su opinión sobre cuestiones referidas a acontecimientos del futuro. Las estimaciones de los expertos se realizan en sucesivas rondas, anónimas, al objeto de tratar de conseguir consenso, pero con la máxima autonomía por parte de los participantes.

Por lo tanto, la capacidad de predicción de la Delphi se basa en la utilización sistemática de un juicio intuitivo emitido por un grupo de expertos.

Es decir, el método Delphi procede por medio de la interrogación a expertos con la ayuda de cuestionarios sucesivos, a fin de poner de manifiesto

\footnotetext{
${ }^{1}$ Para una buena descripción del método Delphi, ver Landeta, Jon. (1999) El método Delphi. Una Técnica de previsión para la incertidumbre. Ariel. Barcelona y Godet, Michel. (1996) Manuel de Prospective Strategique. Dunod. Paris.

${ }^{2}$ Linstone, H., Turoff, M. : « The Delphi Method. Techniques and Applications », Addison-Wesley, 1975, p.3
} 
ESTE

convergencias de opiniones y deducir eventuales consensos. La encuesta se lleva a cabo de una manera anónima (actualmente es habitual realizarla haciendo uso del correo electrónico o mediante cuestionarios web establecidos al efecto) para evitar los efectos de "líderes". El objetivo de los cuestionarios sucesivos, es "disminuir el espacio intercuartil precisando la mediana".

Las preguntas se refieren, por ejemplo, a las probabilidades de realización de hipótesis o de acontecimientos con relación al tema de estudio (que en nuestro caso sería el desarrollo futuro del sector que estamos analizando). La calidad de los resultados depende, sobre todo, del cuidado que se ponga en la elaboración del cuestionario y en la elección de los expertos consultados.

Por lo tanto, en su conjunto el método Delphi permitirá prever las transformaciones más importantes que puedan producirse en el fenómeno analizado en el transcurso de los próximos años.

En la familia de los métodos de pronóstico, habitualmente se clasifica al método delphi dentro de los métodos cualitativos o subjetivos.

Aunque, la formulación teórica del método Delphi propiamente dicho comprende varias etapas sucesivas de envíos de cuestionarios, de vaciado y de explotación, en buena parte de los casos puede limitarse a dos etapas, lo que sin embargo no afecta a la calidad de los resultados tal y como lo demuestra la experiencia acumulada en estudios similares.

Como es sabido, el objetivo de los cuestionarios sucesivos, es "disminuir el espacio intercuartil, esto es cuanto se desvía la opinión del experto de la opinión del conjunto, precisando la mediana", de las respuestas obtenidas. El objetivo del primer cuestionario es calcular el espacio intercuartil. El segundo suministra a 
ESTE

cada experto las opiniones de sus colegas, y abre un debate transdisciplinario, para obtener un consenso en los resultados y una generación de conocimiento sobre el tema. Cada experto argumentará los pro y los contra de las opiniones de los demás y de la suya propia. Con la tercera consulta se espera un todavía mayor acercamiento a un consenso.

De manera resumida los pasos que se llevarán a cabo para garantizar la calidad de los resultados, para lanzar y analizar la Delphi deberían ser los siguientes:

\section{Fase 1: formulación del problema}

Se trata de una etapa fundamental en la realización de un delphi. En un método de expertos, la importancia de definir con precisión el campo de investigación es muy grande por cuanto que es preciso estar muy seguros de que los expertos reclutados y consultados poseen todos la misma noción de este campo.

La elaboración del cuestionario debe ser llevada a cabo según ciertas reglas: las preguntas deben ser precisas, cuantificables (versan por ejemplo sobre probabilidades de realización de hipótesis y/o acontecimientos, la mayoría de las veces sobre datos de realización de acontecimientos) e independientes (la supuesta realización de una de las cuestiones en una fecha determinada no influye sobre la realización de alguna otra cuestión).

\section{Fase 2: elección de expertos}

La etapa es importante en cuanto que el término de "experto" es ambiguo. Con independencia de sus títulos, su función o su nivel jerárquico, el experto será elegido por su capacidad de encarar el futuro y posea conocimientos sobre el tema consultado.

La falta de independencia de los expertos puede constituir un inconveniente; por esta razón los expertos son aislados y sus opiniones son recogidas por vía postal o electrónica y de forma anónima; así pues se obtiene la opinión real de cada experto y no la opinión más o menos falseada por un proceso de grupo (se trata de elimiar el efecto de los líderes). 
Fase 3: Elaboración y lanzamiento de los cuestionarios (en paralelo con la fase 2)

Los cuestionarios se elaborarán de manera que faciliten, en la medida en que una investigación de estas características lo permite, la respuesta por parte de los consultados.

Preferentemente las respuestas habrán de poder ser cuantificadas y ponderadas (año de realización de un evento, probabilidad de realización de una hipótesis, valor que alcanzará en el futuro una variable o evento,...

Se formularán cuestiones relativas al grado de ocurrencia (probabilidad) y de importancia (prioridad), la fecha de realización de determinados eventos relacionadas con el objeto de estudio: necesidades de información del entorno, gestión de la información del entorno, evolución de los sistemas, evolución en los costes, transformaciones en tareas, necesidad de formación,....

En ocasiones, se recurre a respuestas categorizadas (Si/No; Mucho/Medio/Poco; Muy de acuerdo/ De acuerdo/ Indiferente/ En desacuerdo/Muy en desacuerdo) y después se tratan las respuestas en términos porcentuales tratando de ubicar a la mayoría de los consultados en una categoría.

\section{Fase 4: desarrollo practico y explotación de resultados}

El cuestionario es enviado a cierto número de expertos (hay que tener en cuenta las no-respuestas y abandonos. Se recomienda que el grupo final no sea inferior a 25). Naturalmente el cuestionario va acompañado por una nota de presentación que precisa las finalidades, el espíritu del delphi, así como las condiciones prácticas del desarrollo de la encuesta (plazo de respuesta, garantía de anonimato). Además, en cada cuestión, puede plantearse que el experto deba evaluar su propio nivel de competencia.

El objetivo de los cuestionarios sucesivos es disminuir la dispersión de las opiniones y precisar la opinión media consensuada. En el curso de la $2^{a}$ a consulta, los expertos son informados de los resultados de la primera consulta de preguntas y deben dar una nueva respuesta y sobre todo deben justificarla en el caso de que sea fuertemente divergente con respecto al grupo. Si resulta necesaria, en el curso de la 3 a consulta se pide a cada experto comentar los argumentos de los que disienten de la mayoría. Un cuarto turno de preguntas, permite la respuesta definitiva: opinión consensuada media y dispersión de opiniones (intervalos intercuartiles). 
ESTE

ESTE

Facultad de CC. EE. Ekonomia eta Enpresa

y Empresariales

Zientzien Fakultatea

\section{Algunos pasos prácticos}

\section{Grabar los resultados en una base de datos.}

Es un labor que aunque el número de respuestas sea reducido, aproximadamente 25-30, merece la pena ya que permite llevar a cabo correcciones o modificaciones con rapidez.

Obtención de los principales resultados: los principales estadísticos que se emplearán en el estudio serán medidas de tendencia central y dispersión:

\section{Media, mediana, moda, máximo, mínimo y desviación típica.}

Ello nos permite tener una visión de conjunto de los resultados obtenidos en cada una de las preguntas, aunque luego sólo se utilice como valor para la segunda vuelta la media o la mediana.

La media y la mediana nos indica la tendencia central de la distribución o conjunto de respuesta de expertos, al igual que la moda.

El máximo y el mínimo nos indican las respuestas extremas.

La desviación nos señala el grado de dispersión en las respuestas ( si más o menos los expertos se hallan en torno a las cifras de la media o no)

Los cuartiles, vendrían a ayudar también en la visión del grado de dispersión de las respuestas.

\{El quartil 1 (Q1), que es igual al percentil 25, sería el valor que deja el $25 \%$ de las respuestas por debajo de ella y el 75\% por encima. El quartil 3 (Q3), que es igual al percentil 75, sería el valor que deja el 75\% de las respuestas por debajo de ella y el $25 \%$ por encima. Es decir entre Q1 y Q3, se situaría la mitad central de las respuestas obtenidas\}.

Para llevar a cabo estas tareas cualquier programa de tratamiento estadístico e incluso una hoja de cálculo, resulta válida.

Por ejemplo, el programa Excel podría valer para grabar y tener los datos registrados y también obtener las medias, medianas,... de cada una de las cuestiones planteadas en la consulta realizada a las empresas. 
Ejemplo: ¿En qué año el $40 \%$ de las ventas de vinos de calidad (crianzas, reservas, grandes reservas) se realizarán por Internet en España?

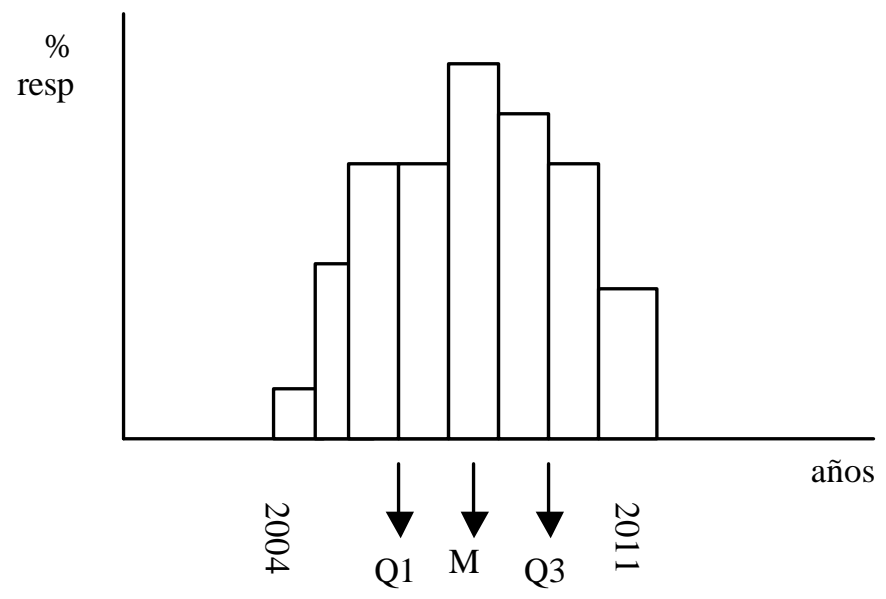

\section{Lanzamiento de la segunda vuelta}

Como puede observarse en la formulación más académica o teórica del método Delphi (Godet 3 , 1996: Landeta 4 , 1999), el trabajar buscando la convergencia de los expertos en base a disminuir el espacio intercuartil precisando la mediana supone el tener que realizar tres o más vueltas (consultas) a los expertos. Por ello, aunque se han llevado a cabo formulaciones clásicas del método Delphi, habida cuenta de los objetivos de la investigación y el horizonte temporal relativamente próximo respecto al que encaramos el estudio prospectivo, optaremos por el siguiente sistema:

\section{Objetivo del lanzamiento y tratamiento de la segunda vuelta}

La realización de una segunda vuelta en el estudio se plantea con un doble objetivo:

3 Godet, Michel. (1996) Manuel de Prospective Strategique. Dunod. Paris.

${ }^{4}$ Landeta, Jon. (1999)El metodo Delphi. Ariel. Barcelona. 
ESTE

ESTE

Facultad de CC. EE. Ekonomia eta Enpresa y Empresariales

Zientzien Fakultatea

a) remitir y hacer participes de la información obtenida a todos aquellos que han colaborado en el estudio con la aportación de su conocimiento y opiniones

b) consolidar y refrendar los resultados obtenidos en la consulta inicial. De hecho, la experiencia indica que las variaciones respecto a los resultados iniciales son mínimas en éste tipo de estudios.

\section{Metodología para el lanzamiento y tratamiento de la segunda vuelta}

a) Se selecciona la media o la mediana de las respuestas a las preguntas de la primera ronda, dependerá del tipo de pregunta, aunque habitualmente, - si las desviaciones típicas no son excesivas - se utiliza la media.

b) Se solicita a los expertos que indiquen su acuerdo o desacuerdo con dicha media.

c) Se pide a los expertos que no se hallan de acuerdo con la media que argumenten sus razones

¿Esta Ud. de acuerdo con la media obtenida para el conjunto de los consultados? Si/no

En caso de que no se halle de acuerdo, ¿Cuál es el nuevo valor que propone?

Si lo juzga necesario, ¿podría justificar dicha respuesta? 
d) Se calcula la nueva media o mediana (a los expertos que se muestren de acuerdo se les fijara el valor de la media anterior).

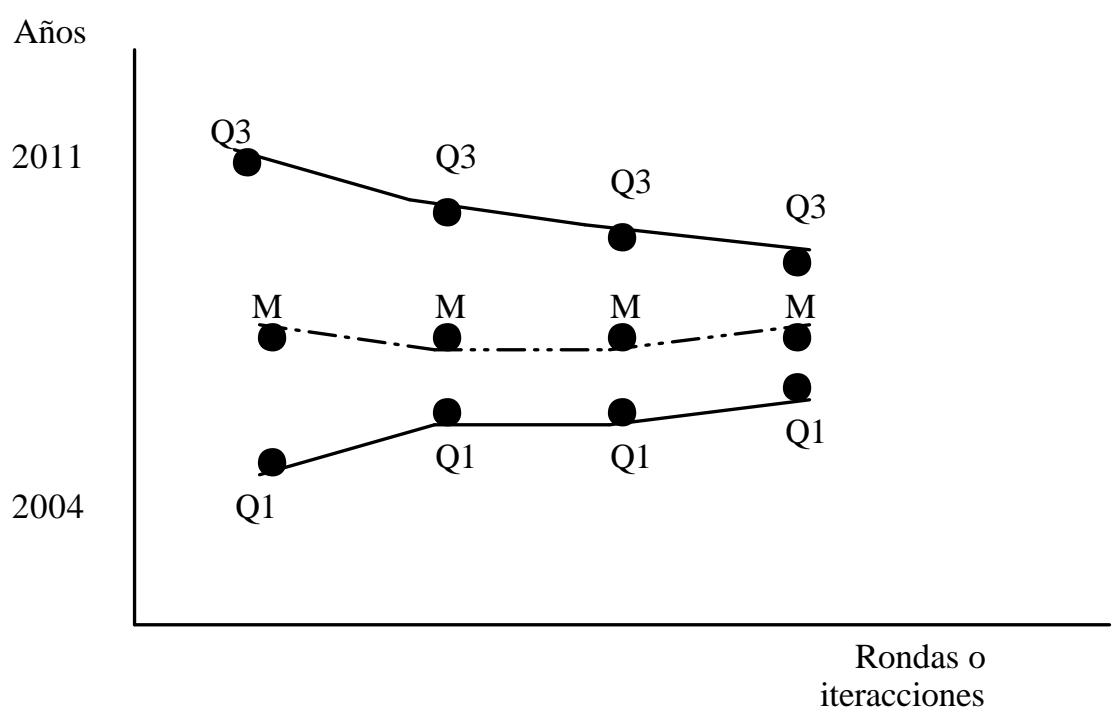

Los valores propuestos y razonados por los expertos que siguen manteniendo valores diferentes de la media, sirven para elaborar escenarios alternativos o formular hipótesis de futuro alternativas (el futuro no es único y predeterminado), que serán más valiosas en función de la "calidad" del experto en el tema. Por ello, es conveniente recoger también los más significativos en el informe que se elabore, haciendo mención:

P.ej. La mayoría de los expertos consultados opinan que la implantación másiva del sistema de información del entorno A se hará patente en el año 2003, aunque algunas opiniones sostienen que no dará de manera significativa en el sector hasta el año 2.006 debido a que .... (incluir la argumentación del experto)

A este respecto, señalar que a veces se ha recurrido calificar la "competencia" del experto en cada pregunta o bloque de preguntas (también se ha utilizado la autocalificación) y a ponderar las respuestas en función de la calificación del 
experto. Muy bueno (3), bueno (2), regular (1), no es competente para responder $(0)$. Sin embargo, dado el carácter del estudio no se considera necesaria ésta medida.

\section{Elaboración de informe}

Los resultados más destacados de la encuesta, las tablas estadísticas de resultados y las incidencias del trabajo de campo se recogen en un informe específico.

\section{Algunas cuestiones adicionales}

\section{Número óptimo de expertos}

Aunque no hay forma de determinar el número óptimo de expertos para participar en una encuesta Delphi, estudios realizados por investigadores de la Rand Corporation 5 , señalan que si bien parece necesario un mínimo de siete expertos habida cuenta que el error disminuye notablemente por cada experto añadido hasta llegar a los siete expertos, no es aconsejable recurrir a más de 30 expertos, pues la mejora en la previsión es muy pequeña y normalmente el incremento en coste y trabajo de investigación no compensa la mejora.

\section{Herramientas on-line}

Con el desarrollo de Internet han comenzado tambien ha desarrollarse herramientas on-line para todo tipo de análisis relacionados con la estrategia y la prospectiva. Así, por ejemplo, la compañía Calibrum (http://www.calibrum.com ), además de ofrecer productos para el desarrollo on-line de procesos de planificación estratégica o la

\footnotetext{
${ }^{5}$ Norman C. dalkey, Bernice Brown y S. Cochran, "The Delphi Method, III: Use of self rating to improve group estimates". Technological Forecasting and Social Change, vol 1, 1970, pp. 283-91. Citado por Landeta, Jon (1999) op. cit.
} 
ESTE

ESTE

Facultad de CC. EE. Ekonomia eta Enpresa

y Empresariales

Zientzien Fakultatea

gestión de proyectos, incluye también una herramienta para el desarrollo de Delphis on-line (http://www.calibrum.com/tf_delphi.htm)

Las herramientas en red dan un soporte tecnológico para la resolución de los problemas: Tiempo, Dinamismo, Información y Participación.

La herramienta Surveylet permite la realización de un cuestionario Delphi on-line. Así, según señalan sus creadores, Surveylet facilita la participación colectiva y retroalimentación dinámica de resultados mediante el uso de encuestas de opinión y consultas Delphi disponibles en Internet, que admiten la posibilidad de reflexionar ante posibles cambios, y procesar inmediatamente la información on-line. Simplifica la toma de decisiones y la consulta colectiva. El acceso a la encuesta se realiza mediante una página pública y la personalización del cuestionario mediante un código de entrada, representa una innovación y mejora en la planificación estratégica y estudios prospectivos.

\title{
Un ejemplo en España. El programa de Prospectiva del OPTI. Observatorio de Prospectiva Tecnológica I ndustrial
}

El Primer Programa de Prospectiva llevado a cabo por el OPTI entre 1998 y 2001 está constituido por un total de 26 estudios realizados en 8 sectores de actividad. Para la ejecución de estos estudios se formarón 26 Paneles de Expertos y se elaboraron otros tantos cuestionarios Delphi que fueron sometidos a la opinión de $\mathbf{5 . 0 0 0}$ especialistas, con un índice de respuesta después de circular dos veces los cuestionario, del $32 \%$.

Según los responsables del OPTI, éste índice avala plenamente la información obtenida y homologa el Programa español con los mejores ejercicios de prospectiva realizados en el ámbito internacional. Los resultados de los cuestionarios Delphi han sido analizados por los Paneles de Expertos, dando lugar a la identificación de tendencias tecnológica y tecnologías críticas asociadas, así como al establecimiento de escenarios de futuro.

El horizonte temporal de estos estudios de prospectiva es de 15 años

Los estudios de prospectiva sectorial realizados hasta el momento han sido:

\begin{abstract}
Agroalimentario Tecnologías de Conservación de Alimentos. La biotecnología aplicada al sector alimentario. Tecnologías en el envasado agroalimentario.
\end{abstract}

Energía Energías Renovables.

Tecnologías avanzadas de conversión de combustibles fósiles. 
ESTE

ESTE

Facultad de CC. EE. Ekonomia eta Enpresa

y Empresariales

Zientzien Fakultatea

Tendencias tecnológicas en transporte, distribución, almacenamiento y uso final de la energía.

Medio Ambiente Industrial

Gestión y Tratamiento de residuos industriales.

Bienes de equipo medio ambientales.

Tratamientos de aguas industriales.

Químico

Química Fina.

Química Básica Orgánica. Primeras Materias Plásticas.

Agroquímica.

Pasta, Papel y Cartón.

Tecnologías de la Información y las Comunicaciones

I ndustria de contenidos digitales.

Las TI C y la emergente economía digital.

Convergencia de infraestructuras y servicios en el sector de las telecomunicaciones.

Transportes

Aeronáutico.

Ferrocarril

Naval.

Automoción.

Sectores Básicos y Transformadores

Tecnologías de Fabricación de Productos Metálicos

Tecnologías de transformación de piezas de plásticos y materiales compuestos

Bienes de equipo para la fabricación de piezas unitarias

Sectores Tradicionales

Tecnologías de Diseño

Tecnologías de automatización

Tecnologías limpias y de reciclaje

Estos estudios están publicados en tres Informes de Prospectiva Tecnológica que pueden ser consultados en el apartado de PUBLICACIONES de la página web del OPTI: http: www.opti.org

\section{ÚTI LI DAD Y LÍ MITES DEL MÉTODO DELPHI}

Una de las ventajas del delphi es la quasi-certeza de obtener un consenso en el desarrollo de los cuestionarios sucesivos (pero jatención! convergencia o consenso no significa coherencia). Por lo demás, la información recogida en el curso de la consulta acerca de acontecimientos, tendencias, rupturas determinantes en la evolución futura del problema estudiado, es generalmente rica y abundante. Finalmente, este método 
ESTE

ESTE

Facultad de CC. EE. Ekonomia eta Enpresa y Empresariales

Zientzien Fakultatea

puede utilizarse indistintamente tanto en el campo de la tecnología, de la gestión y de la economía como en el de las ciencias sociales.

Varios son los problemas que limitan el alcance del método que se revela largo, costoso, fastidioso e intuitivo más que racional. Si bien es cierto que las nuevas tecnologías han permitido el relanzamiento del método Delphi, que ciertamente había caído en cierto desuso. La tramitación presionante (encuesta en varias tandas) es además discutible puesto que solo los expertos que se salen de la norma deben justificar su posición. Sin embargo, podemos considerar también que la opinión de los divergentes es, en terminos de prospectiva, más interesente que aquella de los que entran en el rango. Por otra parte, no se toman en consideración las posibles interacciones entre las hipótesis consideradas y son incluso evitados en la propia construcción de la encuesta, esto es lo que ha conducido a los promotores del método Delphi a desarrollar los métodos de impactos cruzados probabilistas.

\section{CONCLUSI ONES PRÁCTI CAS}

Aparentemente el Delphi parece un procedimiento simple, fácilmente aplicable en el marco de una consulta a expertos. Sin embargo existe el riesgo de que los fracasos y/o decepciones desanimen a los "usuarios aficionados". El método viene bien para las aplicaciones decisionales, pero debe estar adaptada en función del objetivo del estudio para la prospectiva. En particular, no es necesario obtener a toda costa una opinión consensuada mediana, pero es importante poner en evidencia varios grupos de respuestas para el análisis de puntos de convergencia múltiples.

Delphi es sin duda una técnica que desde hace unos cuarenta años ha sido objeto de múltiples aplicaciones en el mundo entero.

A partir del procedimiento original, se han desarrollado otras aproximaciones. De este modo, la mini-Delphi propone una aplicación en tiempo real del método: los expertos se reunen en un lugar y debaten cada cuestión antes de responder. Últimamente, la utilización de nuevos modos de interacción entre expertos, como el correo electrónico, tienden a desarrollarse y a convertir el procedimiento en más flexible y rápido. 


\section{BI BLI OGRAFÍ A COMPLEMENTARI A}

BIGUES P.A., Prospective et compétitivé, Mac Graw Hill 1985.

GODET M., Manuel de prospective stratégique, tome 2: L'art et la méthode, Dunod, Paris 1997.

HELMER O., Looking forward: a guide to futures research, Sage publications, 1983.

LANDETA, Jon. El metodo Delphi. Ariel. 1999. Barcelona.

LINSTONE H.A., TURROF, M., The Delphi method, techniques and applications, Addison wesley publishing, 1975.

MARTINO J.P., Technological forecasting for decision making, Mac Graw Hill, 1993.

SAINT-PAUL R., TÉNIÈRE-BUCHOT P.F., Innovation et évaluation technologiques, Entreprise moderne d'édition, 1974. 